\title{
Effects of an Infection Control Protocol for Coronavirus Disease in Emergency Mechanical Thrombectomy
}

\author{
Jin Eun, Min-Hyung Lee, Sang-Hyuk Im, Won-Il Joo, Jae-Geun Ahn, Do-Sung Yoo, Hae-Kwan Park \\ Department of Neurosurgery, Eunpyeong St. Mary's Hospital, College of Medicine, The Catholic University of Korea, Seoul, Korea
}

Objective : Since the outbreak of the coronavirus disease 2019 (COVID-19) pandemic, neurointerventionists have been increasingly concerned regarding the prevention of infection and time delay in performing emergency thrombectomy procedures in patients with acute stroke. This study aimed to analyze the effects of changes in mechanical thrombectomy protocol before and after the COVID-19 pandemic on procedure time and patient outcomes and to identify factors that significantly impact procedure time.

Methods : The last-normal-to-door, first-abnormal-to-door, door-to-imaging, door-to-puncture, and puncture-to-recanalization times of 88 patients ( 45 treated with conventional pre-COVID-19 protocol and 43 with COVID-19 protection protocol) were retrospectively analyzed. The recanalization time, success rate of mechanical thrombectomy, and modified Rankin score of patients at discharge were assessed. A multivariate analysis was conducted to identify variables that significantly influenced the time delay in the door-to-puncture time and total procedure time.

Results : The door-to-imaging time significantly increased under the COVID-19 protection protocol $(p=0.0257)$ compared to that with the conventional pre-COVID-19 protocol. This increase was even more pronounced in patients who were suspected to be COVID-19-positive than in those who were negative. The door-to-puncture time showed no statistical difference between the conventional and COVID-19 protocol groups $(p=0.5042)$. However, in the multivariate analysis, the last-normal-to-door time and door-to-imaging time were shown to affect the door-to-puncture time ( $p=0.0068$ and 0.0097 ). The total procedure time was affected by the occlusion site, last-normal-to-door time, door-to-imaging time, and type of anesthesia ( $p=0.0001,0.0231,0.0103$, and 0.0207 , respectively).

Conclusion : The COVID-19 protection protocol significantly impacted the door-to-imaging time. Shortening the door-to-imaging time and performing the procedure under local anesthesia, if possible, may be required to reduce the door-to-puncture and doorto-recanalization times. The effect of various aspects of the protection protocol on emergency thrombectomy should be further studied.

Key Words : Thrombectomy · Stroke, acute · COVID-19.

\section{INTRODUCTION}

The coronavirus disease 2019 (COVID-19) pandemic has affected all aspects of human life. The use of protective measures against droplets with high infectivity has become mandatory in all medical fields ${ }^{20}$. Ensuring optimal protection is

- Received : February 24, 2021 •Revised : June 11, 2021 •Accepted : August 17, 2021

- Address for reprints : Hae-Kwan Park

Department of Neurosurgery, Eunpyeong St. Mary's Hospital, College of Medicine, The Catholic University of Korea, 1021 Tongil-ro, Eunpyeong-gu, Seoul 03312, Korea Tel : +82-2-2030-4490, Fax : +82-2-2030-4626, E-mail : parkoct@catholic.ac.kr, ORCID : https://orcid.org/0000-0001-7132-7009 
particularly difficult in emergency medicine because it is not possible to reliably distinguish patients with COVID-19 at the initial examination. Frequently, a number of patients are also diagnosed with asymptomatic COVID-19 infection ${ }^{211}$. Hospitals must implement measures to ensure that every patient is tested to prevent the risk of spreading COVID-19, and the medical staff needs to comply with infection control procedures, including the correct use of personal protective equipment (PPE), in all situations ${ }^{20)}$.

From a neurointerventionist's point of view, it is of great interest how the protective processes affect the time for recanalization of large vessel occlusions and its clinical outcomes. In this study, we compared the mechanical thrombectomy time indices and clinical outcomes of procedures conducted according to the conventional protocol before the COVID-19 pandemic with those of procedures conducted as per the new COVID-19 protection protocol. Furthermore, we tried to determine which variables significantly affected procedure time.

\section{MATERIALS AND METHODS}

This research was approved by the Institutional Review Board of Eunpyeong St. Mary's Hospital, College of Medicine, The Catholic University of Korea (IRB No. : PC21RISI0016) and was conducted in accordance with the Declaration of Helsinki.

\section{Patient population}

Eunpyeong St. Mary's Hospital was established in April 2019. In February 2020, after a transport agent of the hospital was diagnosed with COVID-19, the hospital had to be closed under the direction of the Korean Disease Control and Prevention Agency. The hospital was reopened from March 2020 after the COVID-19 protection protocol was established. We retrospectively analyzed the mechanical thrombectomy outcomes before and after the establishment of the protection protocol. Between April 2019 and December 2020, 109 patients with large vessel occlusion were treated with mechanical thrombectomy for an acute stroke. Of these, 55 were diagnosed between April 2019 and February 2020 and 54 between March 2020 to December 2020. Of the total patients, five were excluded because a stenotic lesion without occlusion was present and only angioplasty was performed. Seven patients were excluded because they had only undergone diagnostic angiography and their occluded vessels were judged to have spontaneously recanalized, or they had chronic occlusions that did not need further intervention. We further excluded nine patients who underwent mechanical thrombectomy during hospitalization. Finally, 88 patients were included in this study who underwent mechanical thrombectomy for large vessel occlusions on an emergency basis. Of these 88 patients, 45 were managed using the conventional protocol and 43 were treated as per the COVID-19 protocol (Fig. 1).

In this study, we investigated the last-normal-to-door time, first-abnormal-to-door time, door-to-imaging time, door-topuncture time, puncture-to-recanalization time, total procedure time, Thrombolysis in Cerebral Infarction (TICI) grade, and modified Rankin score (mRS) at discharge.

\section{Imaging and equipment}

Multiphase computed tomography (CT) angiography (CTA) and perfusion CT (CTP) were performed to identify the location of an occlusion site (SOMATOM Force; Siemens, Erlangen, Germany) to evaluate the extracranial and intracranial vessel lesions. If enhanced imaging was not possible, a non-enhanced brain CT scan and diffusion magnetic resolution image (MRI) with perfusion were recorded with time-offlight MR angiography to detect vessel lesions (MAGNETOM Vida; Siemens). A biplane angiography equipment, Siemens' Artis Q biplane, was used for mechanical thrombectomy.

\section{Conventional protocol vs. COVID-19 protection protocol}

The conventional protocol was applied before the COVID-19 pandemic. When a patient visited the emergency room (ER) of our hospital, an emergency medical doctor first triaged the patient, and a "severe emergency call" was activated if the patient had developed neurological symptoms within the last 24 hours. Simultaneously, tissue plasminogen activator (tPA) was prepared according to the patient's weight, and an intravenous line was secured for CTA. Once the stroke team arrived at the ER, brain images were evaluated, and if the patient had arrived at the hospital within 4.5 hours from the last-normal time, intravenous tPA was administered, except for patients for whom it was contraindicated ${ }^{13)}$.

Mechanical thrombectomy was considered in the cases where anterior circulation (internal carotid artery or middle ca- 


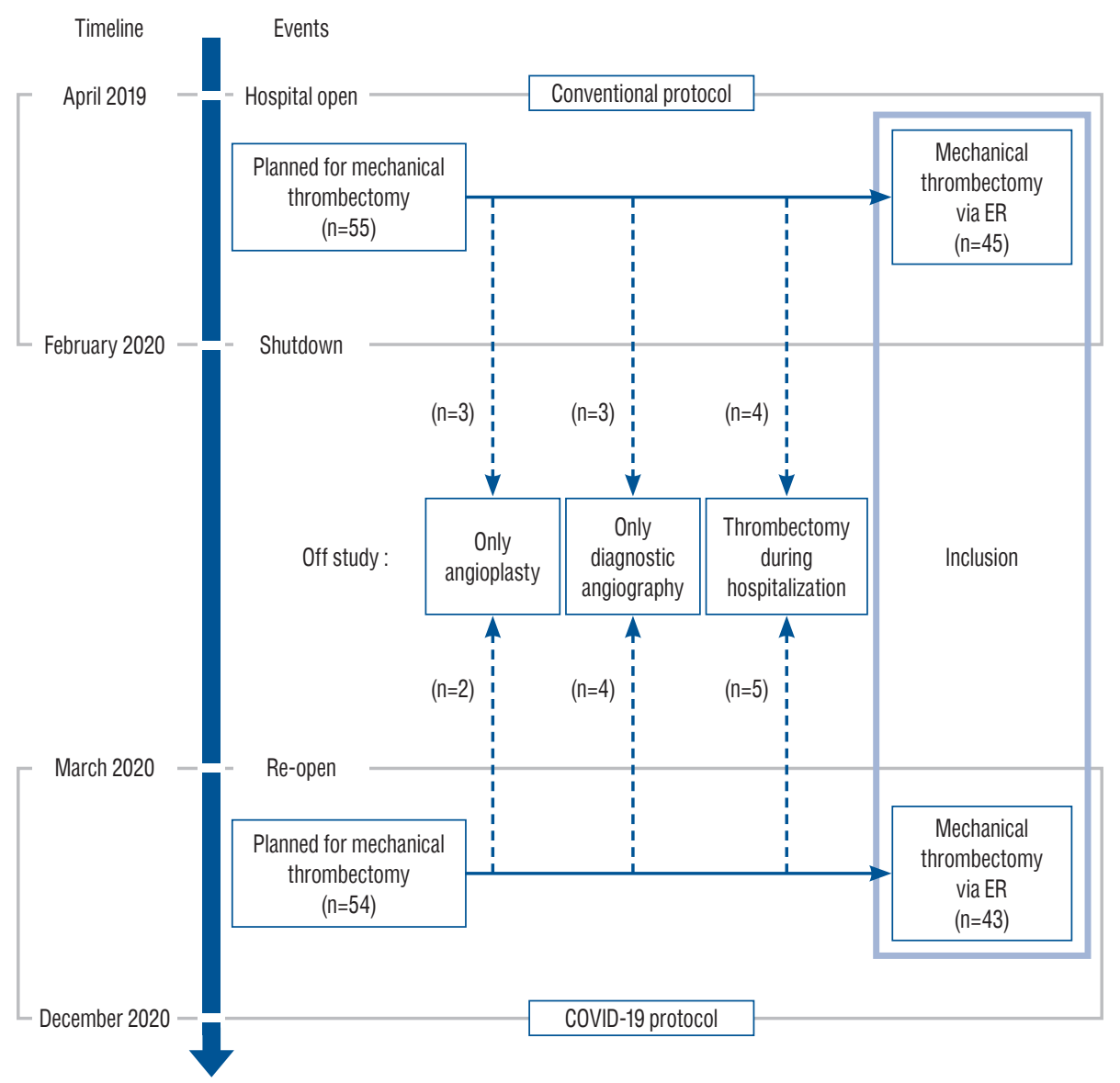

Fig. 1. Timeline and patient selection process before and after establishing the coronavirus disease 2019 (COVID-19) protocol. Before the COVID-19 pandemic, mechanical thrombectomy was conducted for 55 patients. After the hospital reopened, mechanical thrombectomy was conducted for 54 patients. Finally, total 45 patients were treated under the conventional protocol and 43 patients under the COVID-19 protocol were included in this analysis. ER : emergency room.

rotid artery) occlusions or posterior circulation (basilar artery or posterior cerebral artery) occlusions, confirmed as small infarctions with diffusion/perfusion mismatch, occurred within 24 hours or 6 hours from the last-normal time ${ }^{12,13}$.

Diffusion MRI was required to confirm the infarction volume in selective cases. Mechanical thrombectomy was performed in the angiography room or the hybrid operation room depending on the availability of staff. If mechanical thrombectomy was conducted in the hybrid operation room, the interventionist determined whether to apply general anesthesia.

Under the COVID-19 protocol, each patient who arrived at the ER was initially assessed by an emergency medical doctor in the outdoor triage room. Then, the patient was moved to an isolated negative pressure room and photographed using a portable chest radiography machine. Any patient with consol- idation or ground-glass opacity on chest radiograph or upper respiratory symptoms or fever was suspected to be COVID-19 positive (+). Otherwise, the patient was assumed to be COVID-19 negative (-). Even if the patient was assumed to be COVID-19 (-), a confirmative test was conducted. For all patients, an isolated route was used for all subsequent movements, tests, and procedures before the COVID-19 test result was confirmed. A negative pressure tent was used outside the negative pressure room for suspected COVID-19 (+) suspected patients. For suspected COVID-19 (-) patients, a Kf94 (Dr. Puri ${ }^{\circledR}$ KF [Korea filter] 94-fine dust, yellow dust mask; KM Healthcare Corp., Guri, Korea) or N95 (3 M ${ }^{\mathrm{TM}}$ Health Care Particulate Respirator 1860; 3 M Health Care ${ }^{\mathrm{TM}}$, St. Paul, MN, USA) filtering facepiece respirator and a bouffant cap were worn by the treating staff ${ }^{6}$. When patients were taken to the CT room for brain imaging, non-enhanced chest CT was also 
conducted to check for lung lesions as it is more precise than radiography. If a lung lesion that was not seen on the radiograph was found on chest CT, patients were treated as suspected COVID-19 $(+)$ cases thereafter ${ }^{8)}$. Following the new protocol, only CTP was used to evaluate the hypoperfusion area in suspected COVID-19 (+) patients since there was a high chance of time delay for other patients because of the need for complete blockage and cleaning of the MRI room, which usually took more than 30 minutes. To protect the medical staff, our hospital established a PPE system according to the guidelines of the Korean Disease Control and Prevention Agency ${ }^{7}$. When treating suspected COVID-19 (+) cases, level D protection gown with double gloving, protection goggles, and an N95 mask were worn by the medical staff, and a positive air purifying respirator was added during procedures. When treating suspected COVID-19 (-) patients, basic PPE, including a disposable plastic apron, a Kf94 or N95 mask, a surgical cap, nitrile gloves, and a face shield (5-set) were worn by the medical staff ${ }^{7,18)}$. In our facility, the angiography room was located in a separate space with a separate air-conditioning system. In contrast, the hybrid operation room was not isolated from other operating rooms. Therefore, when performing mechanical thrombectomy in patients who were suspected to be COVID-19 (+), only the angiography room was used. If a patient was suspected to be COVID-19 (-), the hybrid operation room was used, but the air-conditioning was blocked to prevent any cross-contamination. Once mechanical thrombectomy was performed, patients were transferred to a neuro-intensive care unit (NCU) via an isolated route under a negative pressure tent if suspected to be COVID-19 (+) or with a Kf94/N95 mask and bouffant cap if suspected to be COVID-19 (-). Patients were kept in a negative pressure isolation room inside the NCU until COVID-19 test results were confirmed. Once a patient was confirmed to be COVID-19 (-), the patient was transferred via the routine route and routine care was provided (Fig. 2).

\section{Statistical analyses}

Patients were classified into two groups according to the protocol used. The baseline characteristics of these groups are listed in Table 1. A chi-square test or Fisher's exact test was used for statistical analysis according to the variable factors of the two groups. A Wilcoxon-Mann-Whitney test was used to compare continuous time variables. Before the comparisons, we evaluated the statistical power of the Wilcoxon-MannWhitney test by the method proposed by Shieh et al. ${ }^{16)}$. Assuming that the time variables of the two groups followed an exponential distribution, the effect size (the probability that the value in the first group is less than the value in the second group) was calculated to be 0.7 . A two-sided comparison was performed with a significance level of 0.05 , and the calculated statistical power was over 0.8 when the number of patients in each group exceeded 40 . When comparing the time variables among the three groups, analysis was conducted using a Kruskal-Wallis test followed by a post hoc Dunn's test with Bonferroni $p$-value adjustment ${ }^{2}$. We also conducted a multivariate analysis to identify the variables that have a significant influence on the procedure time. In the multivariate analysis, linear regression models with log-normal errors and identity link were used ${ }^{10)}$ because we observed that the time variables showed evidence of heteroscedasticity and, among the generalized linear models with various distributions, the log-normal regression models fitted the data properly with the lowest Akaike's information criterion. We set up the multivariate models using a stepwise algorithm ${ }^{9}$, and the $p$-value for each variable was calculated through a likelihood ratio test between the models including and excluding the variable. A variable was included in the model if the $p$-value was less than 0.10 . All statistical analyses were performed using the R software (version 4.0.3; R Foundation for Statistical Computing, Vienna, Austria). All $p$-values were two-sided.

\section{RESULTS}

\section{Baseline characteristics and thrombectomy out- comes}

The age distribution of patients between the two groups was similar ( $p=0.7101$ ), while the proportion of females was higher in the conventional group than in the COVID-19 group ( $48.9 \%$ vs. $27.9 \%, p=0.0716$ ). Compared to that before the pandemic, significantly lower proportion of patients received tPA outside the center after the COVID-19 outbreak (No tPA, tPA at our center, tPA at other centers : $62.2 \%, 20 \%$, and $17.8 \%$, respectively, in the conventional group and $65.1 \%$, $32.6 \%$, and $2.3 \%$, respectively, in the COVID-19 group; $p=0.0405)$. No statistically significant differences were found in the last-normal-to-door time and the first-abnormal-to- 


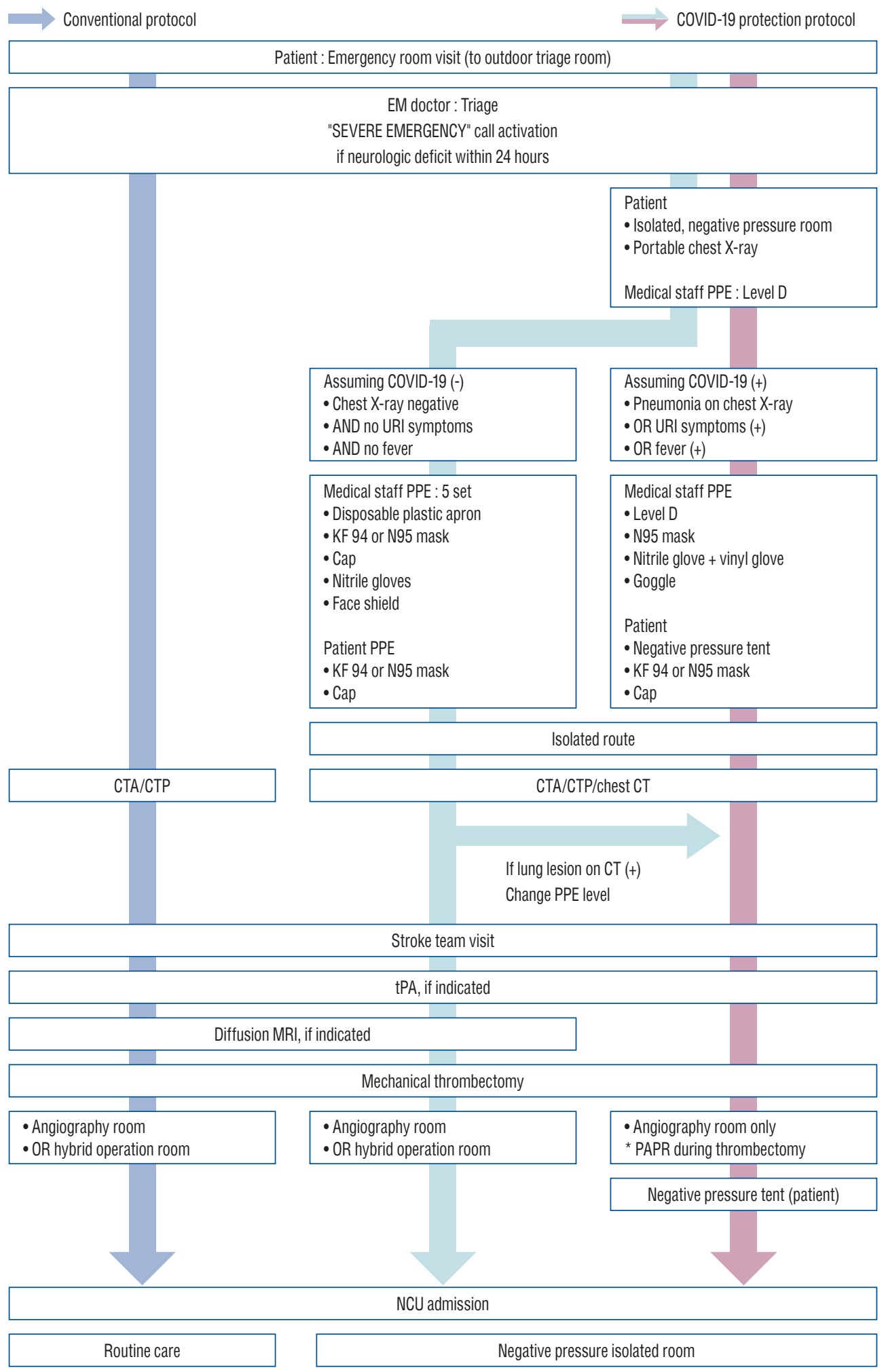

Fig. 2. Comparison of the conventional protocol and COVID-19 protocol. Protection protocol for the prevention of COVID-19 was established and additional steps were included for CT imaging, mechanical thrombectomy, and patient transport route. COVID-19: coronavirus disease 2019, EM : emergency medical, PPE : personal protective equipment, URI : upper respiratory infection, CTA : computed tomography angiography, CTP : perfusion computed tomography, $\mathrm{CT}$ : computed tomography, $\mathrm{PA}$ : tissue plasminogen activator, $\mathrm{MRI}$ : magnetic resolution image, PAPR : powered air purifying respirator, $\mathrm{NCU}$ : neuro-intensive care unit. 
Table 1. Baseline characteristics

\begin{tabular}{|c|c|c|c|}
\hline Protocol & Conventional & COVID-19 & $p$-value \\
\hline Total number & 45 & 43 & \\
\hline Age (years) & $75(62-79)$ & $73(67.5-80.5)$ & 0.7101 \\
\hline Sex & & & 0.0716 \\
\hline Female & $22(48.9)$ & $12(27.9)$ & \\
\hline Male & $23(51.1)$ & $31(72.1)$ & \\
\hline Location & & & 0.1670 \\
\hline ICA & $19(42.2)$ & $9(20.9)$ & \\
\hline M1 & $16(35.6)$ & $21(48.8)$ & \\
\hline M2 & $6(13.3)$ & $6(14.0)$ & \\
\hline Posterior circulation & $4(8.9)$ & $7(16.3)$ & \\
\hline tPA & & & 0.0405 \\
\hline No tPA & $28(62.2)$ & $28(65.1)$ & \\
\hline $\mathrm{tPA}$ & $9(20)$ & $14(32.6)$ & \\
\hline tPA at other centers & $8(17.8)$ & $1(2.3)$ & \\
\hline GCS score & & & 0.1721 \\
\hline$<9$ & $9(20)$ & $4(9.3)$ & \\
\hline $9-12$ & $18(40)$ & $14(32.6)$ & \\
\hline$\geq 13$ & $18(40)$ & $25(58.1)$ & \\
\hline Last-normal-to-indoor time (minutes) & $270(121-504)$ & $262(81.5-511.0)$ & 0.6612 \\
\hline First-abnormal-to-indoor time (minutes) & $57(30-205)$ & $117(42.0-266.5)$ & 0.1406 \\
\hline
\end{tabular}

Values are presented as median (Q1-Q3) or number (\%). COVID-19 : coronavirus disease 2019, ICA : internal carotid artery, M1 : middle cerebral artery, horizontal segment, M2 : middle cerebral artery, insular segment, tPA : tissue plasminogen activator, GCS : Glasgow coma scale

door time between the groups $(p=0.6612$ and 0.1406 , respectively) (Table 1).

In the conventional group, mechanical thrombectomy was more frequently performed in the hybrid operating room than in the angiography room (62.2\% vs. $37.8 \%)$. In contrast, for the COVID-19 group, thrombectomy was more frequently performed in the angiography room than in the hybrid operation room (14.0\% vs. $86.0 \%$ ), and this difference was significant $(p<0.0001)$. Furthermore, mechanical thrombectomy was performed more frequently under local anesthesia in the COVID-19 group than in the conventional group $(83.7 \%$ vs. $31.1 \%$; $p<0.0001$ ). Among the time indices, there was a significant increase in the door-to-imaging time in the COVID-19 group compared to that in the conventional group (median time in the conventional group and COVID-19 group was 14 minutes [interquartile range (IQR), 6-18] and 15 minutes [IQR, 12.0-25.2], respectively; $p=0.0257$ ). Among patients who received tPA, the difference in the door-to-tPA time was borderline between the two groups (median 51 minutes [IQR, 49-57] in the conventional group and 48 minutes [IQR, 45.0$51.8]$ in the COVID-19 group; $p=0.0941)$. It is noteworthy that the door-to-imaging time in the COVID-19 group tended to be longer than that in the conventional group, but no significant difference was observed in the door-to-puncture time between the two groups. The median door-to-puncture time in the conventional and COVID-19 groups was 145 minutes (IQR, 136-162) and 140 minutes (IQR, 107.5-168.0; $p=$ 0.5042 ), respectively. The puncture-to-recanalization time was found to be shorter in the COVID-19 group than in the conventional group (conventional group : median 60 minutes [IQR, 47.0-94.5]; COVID-19 group : median 43 minutes [IQR, 26.5-74.0]; $p=0.0093)$. Moreover, the difference in the total procedure time of the recanalized patients between the groups was borderline significant (median 209 minutes [IQR, 188.0270.5] in the conventional group and 189 minutes [IQR, 158.0-241.5] in the COVID-19 group; $p=0.0580$ ). The postop- 
Table 2. Thrombectomy outcomes according to the protocol

\begin{tabular}{|c|c|c|c|}
\hline Variable & Conventional $(n=45)$ & COVID-19 $(n=43)$ & $p$-value \\
\hline Operation room & & & $<0.0001$ \\
\hline Angiography room & $17(37.8)$ & $37(86)$ & \\
\hline Hybrid room & $28(62.2)$ & $6(14)$ & \\
\hline Anesthesia & & & $<0.0001$ \\
\hline Local & $14(31.1)$ & $36(83.7)$ & \\
\hline General & $31(68.9)$ & $7(16.3)$ & \\
\hline Door-to-imaging time (minutes) & $14(6-18)$ & $15(12.0-25.2)$ & 0.0257 \\
\hline Initial imaging from other hospital & 1 & 3 & \\
\hline Door-to-tPA time in our center (minutes) & $51(49-57)$ & $48(45.0-51.8)$ & 0.0941 \\
\hline Door-to-puncture (minutes) & $145(136-162)$ & $140(107.5-168.0)$ & 0.5042 \\
\hline Puncture-to-recanalization (minutes) & $60(47.0-94.5)$ & $43(26.5-74.0)$ & 0.0093 \\
\hline Failed to recanalization & 2 & 0 & \\
\hline Total procedure time (minutes) & $209(188.0-270.5)$ & $189(158-241.5)$ & 0.0580 \\
\hline Failed to recanalization & 2 & 0 & \\
\hline $\mathrm{TICl}{ }^{*}$ & & & 0.0238 \\
\hline 0 & $2(4.4)$ & $0(0.0)$ & \\
\hline $2 \mathrm{a}$ & $2(4.4)$ & $2(4.7)$ & \\
\hline $2 b$ & $22(48.9)$ & $11(25.6)$ & \\
\hline 3 & $19(42.2)$ & $30(69.8)$ & \\
\hline mRS on discharge & & & 0.1043 \\
\hline $0-1$ & $12(26.7)$ & 8 (18.6) & \\
\hline$\geq 2$ & $33(73.3)$ & $31(72.1)$ & \\
\hline Unknown & $0(0.0)$ & $4(9.3)$ & \\
\hline
\end{tabular}

Values are presented as median (Q1-Q3) or number (\%). *0 : no perfusion, $2 a$ : only partial filling of the entire vascular territory is visualized, $2 b$ : complete filling of all the expected vascular territory is visualized but the filling is slower than normal, 3 : complete perfusion. COVID-19: coronavirus disease 2019, tPA : tissue plasminogen activator, TICl : Thrombolysis in cerebral infarction scale, mRS : modified Rankin scale

erative TICI was superior in the COVID-19 group than in the conventional group (0, 2a, 2b, $3: 0 \%, 4.7 \%, 25.6 \%, 69.8 \%$ in the conventional group and $4.4 \%, 4.4 \%, 48.9 \%, 42.2 \%$ in the COVID-19 group, respectively; $p=0.0238$ ). The patient mRS at discharge were not significantly different between the two groups ( $p=0.1043$ ) (Table 2).

The patients treated under the COVID-19 protocol were classified into two groups : suspected COVID-19 (-) and COVID-19 (+) patients. We compared the door-to-imaging time and door-to-puncture time according to the subgroups (conventional, suspected COVID-19 [-], and suspected COVID-19 [+] groups). In this analysis, we found significant differences in the door-to-imaging time between the three groups ( $p=0.0048$, Fig. 3), with a significant increase in the suspected
COVID-19 (+) group (post hoc $p=0.0043$ and 0.0511 , between the conventional group and suspected COVID-19 [-] group). The median door-to-imaging time in the suspected COVID-19 (+) and COVID-19 (-) groups was 28.5 minutes (IQR, 22-32) and 15 minutes (IQR, 11.2-19.0), respectively. There was no significant difference in the door-to-puncture time between the three groups ( $p=0.7436$ ).

\section{Multivariate analysis of procedure time}

Multivariate analysis was conducted to identify factors that have a significant impact on the door-to-puncture time and total procedure time (Table 3). In our study, the door-topuncture time was significantly influenced by the occlusion site $(p=0.0356)$, the last-normal-to-door time $(p=0.0068)$, 

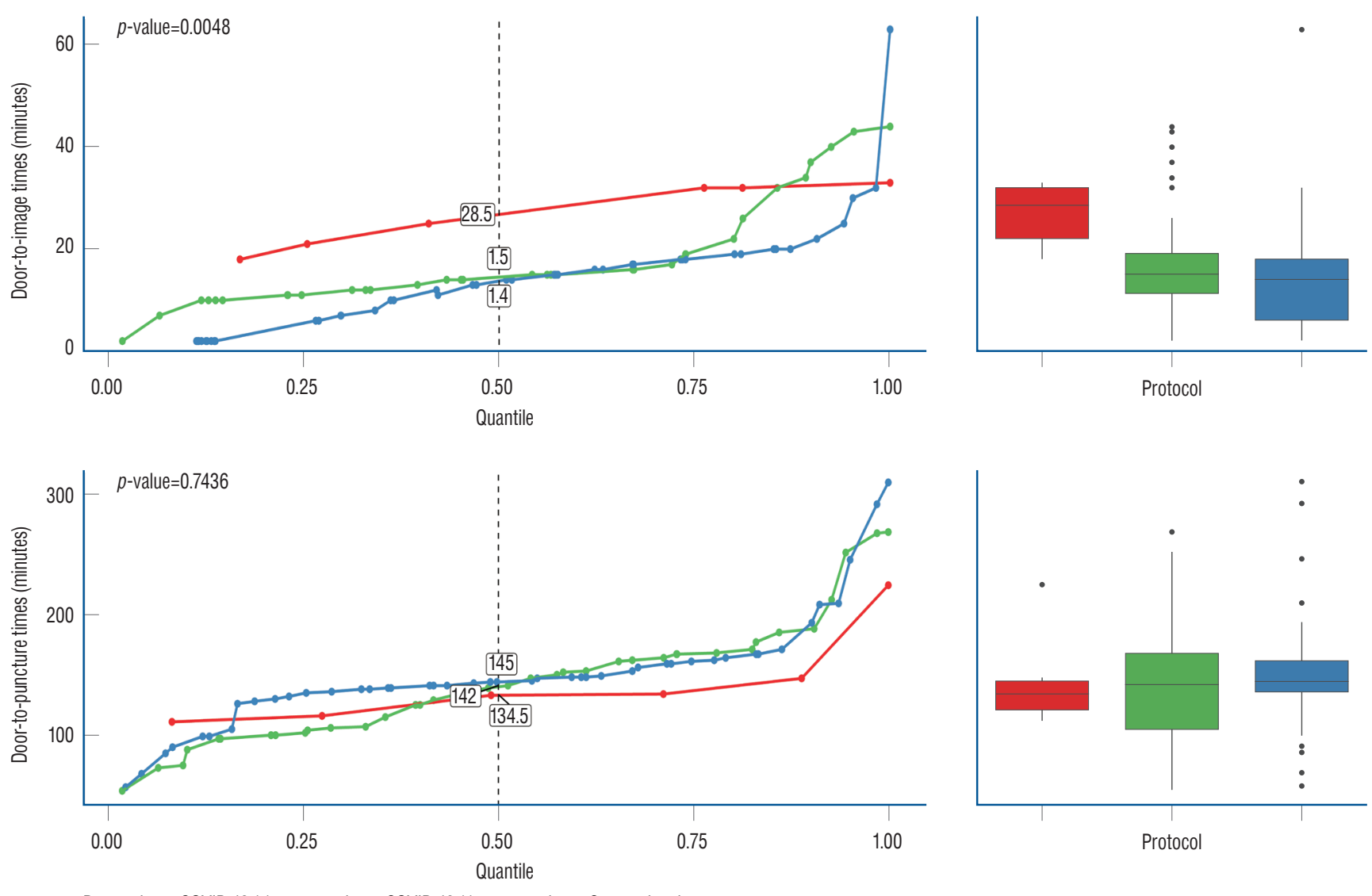

Protocol $\rightarrow$ COVID-19 (+) suspected $\rightarrow$ COVID-19 (-) suspected $\rightarrow$ Conventional

Fig. 3. Graphical comparison of the door-to-imaging time and door-to-puncture time in the conventional, suspected coronavirus disease 2019 (COVID-19) positive, and suspected COVID-19 negative groups. The door-to-imaging time was longer in the suspected COVID-19 positive group compared to that in the suspected COVID-19 negative and conventional protocol groups. The door-to-puncture time showed no statistical difference between the three groups.

Table 3. Multivariate analysis for the door-to-puncture time and total procedure time

\begin{tabular}{|c|c|c|c|c|}
\hline \multirow{2}{*}{ Variable } & \multicolumn{2}{|c|}{ Door-to-puncture time $(n=84)$} & \multicolumn{2}{|c|}{ Total procedure time $(n=84)$} \\
\hline & Parameter* $(95 \%$ Cl) & $p$-value ${ }^{\dagger}$ & Parameter* $(95 \% \mathrm{CI})$ & $p$-value ${ }^{\dagger}$ \\
\hline Occlusion vessel & & 0.0356 & & 0.0001 \\
\hline ICA & $1^{\ddagger}$ & & $1^{\ddagger}$ & \\
\hline M1 & $-16.55(-36.90$ to -3.22$)$ & & $-32.49(-62.37$ to -3.57$)$ & \\
\hline M2 & $-25.83(-50.56$ to -0.10$)$ & & $-59.50(-94.35$ to -23.79$)$ & \\
\hline Posterior circulation & $-35.75(-60.09$ to -9.98$)$ & & $-81.56(-116.05$ to -45.63$)$ & \\
\hline Last-normal-to-door time (per 10 minutes) & 0.31 (0.08 to 0.56$)$ & 0.0068 & $0.36(0.05$ to 0.70$)$ & 0.0231 \\
\hline GCS score & 4.61 (1.51 to 7.65$)$ & 0.0045 & $4.28(-0.14$ to 8.60$)$ & 0.0574 \\
\hline Door-to-imaging time (per 10 minutes) & 11.28 (2.73 to 20.30$)$ & 0.0097 & 15.40 (3.65 to 27.76) & 0.0103 \\
\hline Anesthesia & & 0.0694 & & 0.0207 \\
\hline Local & $1^{\ddagger}$ & & $1^{\ddagger}$ & \\
\hline General & $15.58(-1.26$ to 32.63$)$ & & $28.39(4.45$ to 52.76$)$ & \\
\hline
\end{tabular}

*Parameters are interpreted as the ordinary linear regression model. They indicate average change by one unit increase in each variable. ${ }^{\dagger} p$-values are estimated by the likelihood ratio tests. ${ }^{\ddagger}$ Reference categories. ICA : internal carotid artery, M1 : middle cerebral artery, horizontal segment, M2 : middle cerebral artery, insular segment, GCS : Glasgow coma scale 
Glasgow coma scale (GCS) score at arrival ( $p=0.0045)$, and the door-to-imaging time ( $p=0.0097)$. Type of anesthesia showed borderline significance $(p=0.0694)$. In the model for total procedure time, the same variables were shown to be significant, except GCS score ( $p$-value for occlusion site, last-normal-todoor time, GCS score, door-to-imaging time, and anesthesia type : $0.0001,0.0231,0.0574,0.0103$, and 0.0207 , respectively). Among the hospital-based variables, the door-to-imaging time was the factor that contributed to a significant increase in the door-to-puncture time and total procedure time. The expected increment of the door-to-puncture time per 10-minute increase in the door-to-imaging time was 11.28 minutes (95\% confidence interval [CI], 0.54-17.29), and the expected increase in the total procedure time per 10-minute increase in the door-to-imaging time was 15.4 minutes (95\% CI, 3.6527.76). A significant delay in the total procedure time was observed among patients who received general anesthesia. In our multivariate analysis, expected delay in the total procedure time was 28.39 minutes (95\% CI, 4.45-52.76) in patients who underwent mechanical thrombectomy under general anesthesia when compared to those who received local anesthesia.

\section{DISCUSSION}

Neurointerventionists are under a lot of pressure to timely treat patients with acute stroke, as the saying goes "time is brain" "15). Therefore, for patients with acute stroke requiring mechanical thrombectomy, neurointerventionists must question whether a level of protection that prevents infection but does not significantly affect patient outcomes and time variables is possible. In 2020, Nguyen et al. ${ }^{11)}$ published a mechanical thrombectomy guideline to be used during the COVID-19 pandemic. A few COVID-19-related studies have also discussed protection protocols for managing such cases. However, the protection protocols they proposed were ambiguous and did not provide evidence regarding the extent to which these protective procedures affect care time and outcomes ${ }^{11,20)}$. A previous report by Kerleroux et al. ${ }^{5)}$ analyzed the changes in mechanical thrombectomy outcomes during the COVID-19 pandemic using multi-center data in France. They demonstrated a significant delay in the imaging-to-groin puncture time during the pandemic, which was not seen as significant in patients receiving mechanical thrombectomy at the stroke center $^{5)}$. However, Kerleroux et al. ${ }^{5)}$ did not focus on the detailed correlation between protection protocol and time delay. A recent study by Siegler et al. ${ }^{17)}$ analyzed treatment times of patients with acute ischemic stroke. The authors demonstrated small but significant delay of procedure time in large cohort, and the result is somewhat different from that of our study. However, most of the previous data on these issues are likely to be biased since the data collected before the COVID-19 pandemic would have been collected from medical staff with expertise in the existing conventional protocol, and there is a high possibility that the time indices of the data from the pre-COVID-19 period would be smaller than those in the post-COVID-19 period. Contrarily, the special situation at our center, where a shutdown period was implemented after opening for 1 year, involved a new protocol for the periods before and after the COVID-19 outbreak. This made it possible to objectively evaluate the differences in time delay between the two protection protocols without bias from various aspects.

In the comparison between the conventional and the COVID-19 protocols, the most prominent result was that the patients' door-to-imaging time was significantly longer with the COVID-19 protocol. Further analysis revealed that the significance of this difference was because of the protocol applied for suspected COVID-19 (+) patients, which was distinctively prolonged. The door-to-puncture time could be standardized for all patients if the diffusion MRI step is skipped in all suspected of COVID-19 (+) patients. Though skipping diffusion MRI would result in microbleeds not being detected, CTA with CTP are as effective as diffusion MRI for the detection of penumbra. Therefore, we were able to reduce the time required under the COVID-19 protocol without affecting the efficiency of the protocol ${ }^{4,22)}$.

Interestingly, using a moderate level of protection for suspected COVID-19 (-) patients, there was little difference in time indices from the conventional group. These facts clearly show that the more protection requirements, the greater the time delay. Moreover, it is evident that an appropriate level of protection can be provided with almost no time delay compared to that with the conventional protocol; therefore, a balance between time savings and protection is needed. Based on further understanding of COVID-19 and its diagnosis, it would be beneficial to develop comprehensive criteria to distinguish the patients who require high-level protection from those who do not. An adequate level of protection could be 
provided to other patients if these high-risk patients can be optimally identified, and the same time indices can be achieved as those before COVID-19. Discussions regarding the degree of protection and methods for screening patients with a high likelihood of COVID-19 infection in an emergency setting are warranted.

Through our multivariate analysis, it was confirmed that the significant hospital-based factors to be considered for the door-to-puncture time and total procedure time were the door-to-imaging time and anesthesia type. Considering that the intensive COVID-19 protocol significantly prolonged the door-to-imaging time, as mentioned above, the results of the multivariate models also indicated that setting an appropriate level of protection to reduce the door-to-imaging time is important and must be considered when establishing a protection protocol. Interventionists always consider risk to benefit ratio of mechanical thrombectomy for patients with a long last-normal-to-door time. Additionally, patients need to be informed regarding the hemorrhagic transformation risks after mechanical thrombectomy. These factors may affect the door-to-puncture time and need more specific analysis in future $^{12,14)}$. Based on the anesthesia types, it was confirmed that the use of general anesthesia caused a considerable delay. Recent studies have not been able to prove the superiority or inferiority of one anesthesia type over the other ${ }^{1)}$. One may prefer general anesthesia, given the advantages of patient's airway maintenance and ease of irritability control and the potential risks of conversion from local anesthesia to general anesthesia. However, under the COVID-19 protection protocol, there is already a time delay in obtaining brain imaging before the procedure. Therefore, efforts to reduce the time taken to perform other processes are needed. Furthermore, it is worth noting that the emergency conversion to general anesthesia from local anesthesia in mechanical thrombectomy is reported to be tolerable, and the risk of COVID-19 droplet infection during general anesthesia because of intubation has already been reported ${ }^{3,11}$. Therefore, in situations with high risks of infective diseases, such as COVID-19, it may be a good idea to use local anesthesia first.

The protection protocol is not a small burden for the medical staff, especially for interventionists. Many factors contribute to the burden, including the delayed time caused by the protection processes, increased fatigue by wearing heavy PPE and lead protection equipment simultaneously, and increased difficulty when performing the procedure because of the discomfort caused by PPE; these are difficult to objectively evaluate but cannot be overlooked. In our data, the total procedure time was not prolonged even with a significant increase in the door-to-imaging time because our puncture-to-recanalization time was significantly shortened in patients under the COVID-19 protocol. This was largely attributed to a large portion of patients undergoing mechanical thrombectomy under local anesthesia under the COVID-19 protection protocol. However, there was a possibility of increased burden or fatigue for the medical staff, which may have made mechanical thrombectomy faster. Nevertheless, our data showed that there was no significant difference in patients' $\mathrm{mRS}$ between the two protocols at the time of discharge and that postoperative TICI grades tended to be slightly better in patients in the COVID-19 group than in the conventional group. While various aspects must be considered, it can be argued that the COVID-19 protection protocol did not significantly diminish effectiveness mechanical thrombectomy. There must be further investigations into the impact of the protection protocol from an interventionist's perspective.

This study had several limitations. Even though the size of our data was not exceedingly small, there were only six patients in the suspected COVID-19 (+) group; therefore, sufficient statistical power could not be achieved in the analysis of this group. In addition, since there was no patient who was actually diagnosed with COVID-19 based on the confirmatory test, the actual protection capabilities of our protection protocol could not be evaluated. Furthermore, the effectiveness of classifying a patient as suspected COVID-19 (+) case using only early clinical symptoms and chest radiography with chest CT needs to be justified ${ }^{8,19)}$. Lastly, though we presented a well-organized COVID-19 protection guideline for mechanical thrombectomy, there may be limitations in applying the same protection protocol for other procedures and other hospitals. Recently, many people have been vaccinated against COVID-19. Our hospital is establishing a fast-track protocol for patients who have been vaccinated. We intend to conduct further research after establishing this protocol.

\section{CONCLUSION}

For mechanical thrombectomy, protection protocols to pre- 
vent the spread of COVID-19 affected the time interval from patient arrival to acquiring brain images, particularly in patients needing a high-level of protection. Even though there was no statistical difference in the door-to-puncture time between the conventional and COVID-19 groups, shortening of the door-to-imaging time and performing the procedure under local anesthesia whenever possible are required for shortening of the door-to-puncture and door-to-recanalization times. However, we could not find any evidence indicating inferiority of mechanical thrombectomy outcomes under the COVID-19 protection protocol compared to those conducted under the conventional protocol. The design and processes of a protection protocol that can satisfy both time efficiency and adequate level of protection warrant further in-depth discussion. A protection protocol that is both time efficient and provides an adequate level of protection warrants further indepth investigation.

\section{CONFLICTS OF INTEREST}

No potential conflict of interest relevant to this article was reported.

\section{INFORMED CONSENT}

This type of study does not require informed consent.

\section{AUTHOR CONTRIBUTIONS}

\author{
Conceptualization : JE, HKP \\ Data curation : MHL, SHI, WIJ, JGA, DSY \\ Formal analysis : JE \\ Methodology : WIJ, JGA, DSY \\ Project administration : JE \\ Visualization : MHL, SHI \\ Writing - original draft : JE \\ Writing - review \& editing : HKP
}

\section{ORCID}

$\begin{array}{ll}\text { Jin Eun } & \text { https://orcid.org/0000-0003-4222-7505 } \\ \text { Min-Hyung Lee } & \text { https://orcid.org/0000-0002-2117-8022 } \\ \text { Sang-Hyuk Im } & \text { https://orcid.org/0000-0002-4159-2781 } \\ \text { Won-Il Joo } & \text { https://orcid.org/0000-0002-4120-1699 } \\ \text { Jae-Geun Ahn } & \text { https://orcid.org/0000-0002-1297-7420 } \\ \text { Do-Sung Yoo } & \text { https://orcid.org/0000-0003-2569-6502 } \\ \text { Hae-Kwan Park } & \text { https://orcid.org/0000-0001-7132-7009 }\end{array}$

\section{References}

1. Businger J, Fort AC, Vlisides PE, Cobas $M, A k c a ~ O$ : Management of acute ischemic stroke-specific focus on anesthetic management for mechanical thrombectomy. Anesth Analg 131 : 1124-1134, 2020

2. Dunn OJ : Multiple comparisons using rank sums. Technometrics 6 : 241-252, 1964

3. Flottmann F, Leischner $H$, Broocks G, Faizy TD, Aigner A, Deb-Chatterji M, et al. : Emergency conversion to general anesthesia is a tolerable risk in patients undergoing mechanical thrombectomy. AJNR Am J Neuroradiol 41 : 122-127, 2020

4. Jenson M, Libby J, Soule E, Sandhu SJ, Fiester PJ, Rao D : CT perfusion protocol for acute stroke expedites mechanical thrombectomy. Cureus 11 : e4546, 2019

5. Kerleroux B, Fabacher T, Bricout N, Moïse M, Testud B, Vingadassalom $S$, et al. : Mechanical thrombectomy for acute ischemic stroke amid the COVID-19 outbreak: decreased activity, and increased care delays. Stroke 51 : 2012-2017, 2020

6. Kim MC, Bae S, Kim JY, Park SY, Lim JS, Sung M, et al. : Effectiveness of surgical, kf94, and n95 respirator masks in blocking SARS-CoV-2: a controlled comparison in 7 patients. Infect Dis (Lond) 52 : 908-912, 2020

7. Korean Disease Control and Prevention Agency : New COVID-19 infection prevention and management. Available at : https://kdca. go.kr/board/board.es?mid=a20507020000\&bid=0019

8. Korean Society of Radiology; Guideline Committee; COVID-19 Sub-Committee, Jin KN, Yoon SH, Park CH, Beck KS, Do KH, et al. : KSR/KSTR guidelines for the use of diagnostic imaging for COVID-19. J Korean Soc Radiol 81 : 577-582, 2020

9. Miller $A$ : Subset selection in regression. Boca Raton : Chapman and Hall/CRC, 2002

10. Muggeo $V:$ A note on regression with log normal errors: linear and piecewise linear modelling in R. Available at : https://www. researchgate.net/publication/326319616

11. Nguyen TN, Abdalkader M, Jovin TG, Nogueira RG, Jadhav AP, Haussen $D C$, et al. : Mechanical thrombectomy in the era of the COVID-19 pandemic: emergency preparedness for neuroscience teams: a guidance statement from the Society of Vascular and Interventional Neurology. 
Stroke 51 : 1896-1901, 2020

12. Nogueira RG, Jadhav AP, Haussen DC, Bonafe A, Budzik RF, Bhuva P, et al. : Thrombectomy 6 to 24 hours after stroke with a mismatch between deficit and infarct. N Engl J Med 378 : 11-21, 2018

13. Powers WJ, Rabinstein AA, Ackerson T, Adeoye OM, Bambakidis NC, Becker $\mathrm{K}$, et al. : Guidelines for the early management of patients with acute ischemic stroke: 2019 update to the 2018 guidelines for the early management of acute ischemic stroke: a guideline for healthcare professionals from the American Heart Association/American Stroke Association. Stroke 50 : e344-e418, 2019

14. Saposnik G, Menon BK, Kashani N, Wilson AT, Yoshimura S, Campbell $B C V$, et al. : Factors associated with the decision-making on endovascular thrombectomy for the management of acute ischemic stroke. Stroke $50: 2441-2447,2019$

15. Saver JL: Time is brain--quantified. Stroke 37 : 263-266, 2006

16. Shieh $\mathrm{G}$, Jan $\mathrm{Sl}$, Randles $\mathrm{RH}$ : On power and sample size determinations for the Wilcoxon-Mann-Whitney test. J Nonparametric Stat 18 : 33-43, 2006

17. Siegler JE, Zha AM, Czap AL, Ortega-Gutierrez S, Farooqui M, Liebeskind DS, et al. : Influence of the covid-19 pandemic on treatment times for acute ischemic stroke: The society of vascular and interventional neurology multicenter collaboration. Stroke 52 : 40-47, 2021

18. Sommerstein R, Fux CA, Vuichard-Gysin D, Abbas M, Marschall J, Balmelli C, et al. : Risk of SARS-CoV-2 transmission by aerosols, the rational use of masks, and protection of healthcare workers from COVID-19. Antimicrob Resist Infect Control 9 : 100, 2020

19. The Roya College of Radiologists : The role of CT in screening elective pre-operative patients: 14 May 2020. Available at : https:// www.rcr.ac.uk/college/coronavirus-covid-19-what-rcr-doing/clinicalinformation/role-ct-chest/role-ct-screening-0

20. World Health Organization : Personal protective equipment for COVID-19. Available at : https://www.who.int/teams/health-productand-policy-standards/access-to-assistive-technology-medical-devices/ medical-devices/priority-medical-devices-for-covid/ppe-covid

21. World Health Organization : Report of the WHO-China joint mission on coronavirus disease 2019 (COVID-19). Available at : https://www.who.int/docs/default-source/coronaviruse/who-china-jointmission-on-covid-19-final-report.pdf

22. Yu W, Jiang WJ : A simple imaging guide for endovascular thrombectomy in acute ischemic stroke: from time window to perfusion mismatch and beyond. Front Neurol $10: 502,2019$ 